\title{
AGE-RELATED MACULAR DEGENERATION -AN EMERGING AND RE-EMERGING EYE BLINDING DISEASE
}

\author{
BY
}

\author{
ESENWAH, E. C. \\ DEPARTMENT OF OPTOMETRY \\ FEDERAL UNIVERSITY OF TECHNOLOGY, OWERRI \\ Email: esenwahemma_eye@yahoo.com
}

\begin{abstract}
Age-related macular degeneration (ARMD) is an ocular condition that affects older adults (50years and above age group), resulting in loss of central vision. It is emerging as the leading cause of legal blindness in the world, overtaking glaucoma and diabetic retinopathy combined. A recent study carried out in an Eye Clinic in Owerri over a period of 12 months, using a sample population of 2415 , gave an insight of the prevalence of ARMD .The result of the study is presenting ARMD as an emerging public health concern hence this review.
\end{abstract}

KEYWORDS: Age-related macular degeneration, Retinal pigment epithelium,

\section{INTRODUCTION}

The Macular Disease Society ${ }^{1}$ defined AgeRelated Macular Degeneration (ARMD) as "a primary disease of the Retinal Pigment Epithelium (RPE) with secondary degenerative effect upon the overlying retina and underlying choroidal vessel." Thus, it is a progressive disorder of the central part of the retina called the macular which leads to the gradual loss of central vision needed for reading, writing, driving, watching television, recognizing faces and doing other fine tasks ${ }^{2,3}$.

ARMD is emerging as one of the leading causes of blindness. Indeed, it is the leading cause of blindness in older North Americans, overtaking glaucoma and diabetic retinopathy combined ${ }^{1,4}$. It has also been established as the leading cause of low vision in Americans. The Beaver Dam Eye Study has shown that $30 \%$ of individuals aged 75 and older have some form of ARMD, while $7 \%$ of those 75 and older have advanced form ${ }^{5}$. Recent studies show that about 8million Americans are believed to be at risk of developing ARMD in the next five years, and 1.75 million are currently affected with the advanced form of the disease $e^{6,7}$. Owing to the rapidly aging population, the number of persons having ARMD will increase by $50 \%$ to 2.95 million in $2020^{6}$.

In the United Kingdom, recent studies also show that ARMD is the commonest cause of severe visual impairment. About 1 in 100 people aged 6575years and 1 in 8 people aged 85years and above, suffer from ARMD ${ }^{1}$. The UK statistics also revealed that twice as many women over age of 75years suffer from ARMD compared to men of same age group ${ }^{1}$.

And in Owerri, Nigeria, a recent clinic based survey revealed that 1 in 200 of the adult population aged 50years and above, suffer from one form of ARMD in one or both eye(s). The ratio of distribution was about 3 males to 4 females. The survey also showed that the older you are, the more likely you are to develop ARMD ${ }^{5}$. The above clinical spectra underscored the need for this review.

\section{TYPES OFARMD}

There are two types, the Dry ARMD and the Wet ARMD. The Dry ARMD, also known as the non- neovascular or non-exudative ARMD is the commonest type and accounts for about $85-90 \%$ of all ARMD ${ }^{2,9}$. It is characterized by the gradual thinning, degeneration and eventual death of the RPE of the macular, leading to central visual loss as a result of the breakdown of the photoreceptors of rods and cones ${ }^{2,9}$. The visual loss is gradual. About $20 \%$ of dry ARMD can progress to wet ARMD ${ }^{1,4,9}$.

The wet ARMD, also known as the neovascular or exudative ARMD accounts for about $10-15 \%$ of all cases of ARMD ${ }^{2,6}$. It is the most destructive and the onset can be very rapid. It causes loss of central vision within a short period of time ${ }^{2,9}$. Wet ARMD is caused by abnormal tiny blood vessels that grow in the choriocapillaries which break through the Bruch's membrane and into the macular, leading to blood and protein leakages below the macular. This ultimately results 
to irreversible damage to the photoreceptors of rods and cones. It is believed that the diseased (dead) retina stimulates the introduction of these new blood vessels in response to a decreased supply of nutrient and slow transport of wastes ${ }^{2,9}$.

RISK FACTORS: These include -

Age: ARMD can occur during the middle age but the risk increases with age. Studies show that people in their 50s have about $2 \%$ chance of developing ARMD, while those 60years and above, have $30 \%$ chance of getting it ${ }^{2}$.

Gender: Women are more at greater risk than men $^{1,2}$.

Smoking: Smokers are at risk 2-3 times compared to non-smokers. Smoking lowers the serum antioxidant level in the blood leading to nutritional deficiency at the cellular level. Passive smoking is equally just as bad $^{1,2}$.

Family History: ARMD is not a straightforward hereditary condition as some families may be affected while others may not. The lifetime risk of developing late-stage ARMD is 50\% for people whose relatives have macular degeneration and $12 \%$ for people whose relatives do not have macular degeneration. In addition, people whose relatives have late-stage ARMD also run the risk of developing the disease at a relatively young age $\mathrm{e}^{2,11}$.

Drusen: Drusens are similar in molecular composition to plaques and deposits seen in other age-related diseases such as Alzheimer's disease and Atherosclerosis ${ }^{12,13}$. While drusen deposits may be present in the retina without any visual loss, it has however been blamed for progressive loss of vision. Though the exact role of drusen in the pathogenesis of ARMD is still unclear, it is believed that the lipid content of drusen and its diffuse accumulation in the Bruch's membrane of the retina may be responsible for this loss of vision $^{6,9}$. Recent researches suggest that large and soft drusen are related to elevated cholesterol deposits 9

Cholesterol: Obese and people with elevated levels of blood cholesterol may be at higher risk ${ }^{6}$.

Exposure to Sunlight: People who stay outdoors in the summer sun for more than 5 hours a day in both their teens and 30s are twofold more likely to develop early macular degeneration. Blue light and ultra violet rays have also been reported to increase macular degeneration ${ }^{9,10}$.
High Blood Pressure: People whose blood pressure was about 160/95 before being controlled and those with uncontrolled blood pressure of $160 / 95$ and above, are at risk of developing wet macular degeneration two and three times respectively, compared with people with normal blood pressure. A high pulse pressure has also been associated with wet macular degeneration ${ }^{1,2}$.

Race: Whites are much more at risk than Blacks ${ }^{1}$.

Oxidative Stress: Age-related accumulation of low-molecular-weight phototoxic, pro-toxic melanin oligomers within the lysosomes in the retinal pigment epithelium are suspected of decreasing the digestive rate of photoreceptors of the outer rod segments leading to lipofusion formation associated with macular degeneration".

Other Risk Factors; These include Macular degeneration gene, Stargardt's disease (Juvenile macular degeneration), Fibulin-5 mutation among others ${ }^{9}$.

SYMPTOMS OF ARMD: The commonest early symptoms of ARMD include:

Blurring of Central Vision: The person realizes that he/she needs more light than usual to read and perform other fine tasks. There is difficulty in recognizing faces unless they come close to you ${ }^{1,2}$.

Scotoma; A blind spot may appear in the center of the person's vision. This tends to increase as more rods and cones degenerate in the macular ${ }^{1,2}$.

Visual distortions (metamorphopsia): For wet ARMD, the early sign is when a grid of straight lines appears wavy, e.g. graph paper or the lines between wall tiles ${ }^{1,2}$.

Loss of contrast sensitivity/color perception: There is loss of color perception, ability to trace colors and shadows. Specifically, there is difficulty in differentiating dark colors from dark colors and light ones from light ones ${ }^{1,2,9}$.

Slow recovery of visual function: This usually occurs after exposure to bright light ${ }^{9}$.

\section{DETECTION/DIAGNOSIS}

ARMD is usually detected during eye examination that involves (i) Visual acuity test (ii) Amsler grid test (iii) Dilated fundoscopy (iv) Fluorescein Angiography

*Amsler grid is a pattern of intersecting lines 
identical to a graph paper but with a black or red dot at the center. The central spot is the fixation point. For a normal eye, all lines surrounding the spot will look straight and evenly spaced. In the presence of any macular disease, the lines will look bent, distorted and or missing.

*Dilated fundoscopy may reveal any of the followings:-

(a) Hard drusen- which are small, round, discrete, yellowish-white spots. Patients with hard drusen are at risk of developing geographic atrophy.

(b) Soft drusen- usually larger than hard drusen have indistinct edges. They increase the risk of developing exudative ARMD .

(c) Mixed drusen- refers to the eye with both hard and soft drusen.

(d) Calcified drusen- is a drusen with glistening appearance as a result of dystrophic calcification. They are usually associated with hard drusen.

(e) Basal laminar (nodular) drusens- are innumerable, small, uniformly sized, discrete, round, slightly raised, subretinal lesions, and common in younger people.

\section{Treatment}

Presently, there is no known cure for dry ARMD, apart from taking dietary supplements. For wet ARMD, treatment may halt or delay the progression of vision loss. Indeed, most recent treatment regimens approved by the National Eye Institute (NEI) in America seem to be able to reverse some of the visual losses caused by wet ARMD. The available treatment options include:-

\#Photocoagulation: This involves firing a fine laser directly onto the leaking tiny abnormal blood vessels and burning them off. This procedure may prevent the condition from getting worse but will not restore lost vision. Photocoagulation is suitable only when the leaking blood vessels have not reached or involved the fovea as the laser may also damage otherwise healthy rods and cones ${ }^{1,2,7}$.

\#Photodynamic Therapy: This is a new type of laser treatment. It is far less damaging than photocoagulation. It involves the injection of a photosensitive drug called Verteporfin into the vein of the $\operatorname{arm}^{1,2,7}$. The drug is then activated when light of a specific wavelength, is shone into the eye for about a minute. This leads to the destruction of the abnormally growing blood vessels while sparing the nearby rods and cones. Despite its high success rate, the treatment needs to be repeated every few months, so as to continue to suppress the growth of new blood vessels. This therapy is not suitable for all cases of wetARMD ${ }^{1,2,7}$.

Drugs (Anti-VEGF): Vascular Endothelial Growth Factor (VEGF) is the chemical responsible for the formation of new blood vessels in the macular. Thus, Anti-VEGF drugs block the action of this chemical thereby preventing the formation of the abnormal blood vessels responsible for wet ARMD $^{1,2,7,9}$. These exciting new drugs are also known as Anti-Angiogenics

Anti-VEGF drugs include Ranibizumad (Lucentis), Pegaptanib (Macugen) and Bevacizumad (Avastin). They are injected directly into the vitreous by a fine needle. They are presently giving good results for all stages of wet ARMD. For example, out of the 716 people treated in October, 2006 in New England with Ranibizumab, 9 in 10 people had slowed visual loss, while about a third had improved vision ${ }^{1}$. The only limitation is that the injection could be painful. In addition, it has to be repeated monthly.

The initial aim of treatment with Anti-VEGF was to prevent wet ARMD from getting worse. But this aim seems to have been surpassed, because in some cases these drugs have actually restored some of the vision that had earlier been lost ${ }^{1}$.

Diet/Dietary Supplements: A recent study called "Age- Related Eye Disease Study", (AREDS), showed that some cases of ARMD responded well to dietary supplements containing antioxidants such as vitamins A, C, E, Beta- carotene, including the minerals zinc and copper ${ }^{1,6}$. Lutein and Zeaxanthin have also been shown to slow down macular degeneration. These vitamins and minerals are found in dark green leafy vegetables like spinach and orange bell peppers (Zexthin). They are also found in turkey, chicken and cold water fish ${ }^{1,6,9}$.

While recommending dietary supplements, be mindful of the high risk of increased lung cancer in smokers due to high intake of Beta-carotene, and increased risk of heart failure, in at-risk population, who consume high levels of vitamin E supplements'. One should also be mindful of supplements having only 'window dressing' amounts of specific nutrients. Many companies add tiny amounts of popular nutrients in their products just to be able to put them on their label. Indeed, these little amounts are of no nutritional value $^{6}$.

High fat intake has been implicated as a risk factor in ARMD in both men and women. A diet of $20-25 \%$ total food energy from fat is healthy. Beyond this, no! To reduce fat intake to this level means cutting down on the consumption of red 
meat and high fat dairy products such as butter, cheese, whole milk and others. Eating more of cold water fish and any type of nuts have been known to slow down and even improve the vision of ARMD patients ${ }^{6,9}$.

Resveratrol is a powerful anti- oxidant that has been proved to have many health benefits including ocular health. Thus, moderate drinking of red wine containing high concentration of Resveratrol has been reported to have a positive effect on macular degeneration ${ }^{6}$. Walnuts are also good sources of Resveratrol. The same has been reported for curry souse used in cooking ${ }^{6}$. A recent research revealed that Resveratrol, green tea and curcumin, all have the characteristics to inhibit new fragile blood vessel growth (anti-VEGF) ${ }^{6}$ It has been shown that less salt intake reduces the deposition of waste products in the macular called drusen. Less salt intake also reduces blood pressure and fluid retention ${ }^{6}$.

\section{CONCLUSION/HEALTH INFORMATION/ EDUCATION}

Two thousand, four hundred and fifteen (2415) subjects aged 50 years and above were involved in the Owerri based study ${ }^{5}$. Two hundred and sixtyone $(10.81 \%)$ of them had one form of ARMD in one or both eyes. Males accounted for $45.97 \%$ and females $54.03 \%$. Dry ARMD accounted for $75.86 \%$ and Wet ARMD for $24.14 \%$. While $52.02 \%$ of the Dry ARMD sufferers had visual acuity of 6/36 or less, sufferers of Wet ARMD were $78 \%$. Finally, $6.90 \%$ of ARMD were seen in the 5055 age-group, $13.03 \%$ in the $55-60,18.01 \%$ in the $60-65,27.20 \%$ in the $65-70$ and $34.87 \%$ in the 70 and above age-groups

The above clinical spectra may have been worse if this survey was conducted in a 'free-forservice' rather than in a 'fee-for-service' clinic. Consequently, there is the need for health information and education on ARMD in this country. This would afford the populace including our Health Authorities, the opportunity to appreciate the enormity of this burden, now considered as an emerging eye blinding disease in the world. Certainly, loss of central vision profoundly affects one's life. Early detection and management are the keys to damage limitation.

\section{REF E R E N C E S}

1. Age-related Macular Degeneration Interim Guidelines: Age-related macular degeneration Interim Guideline Royal College of Ophthalmologists (2007).

2. Jong, P. T. (2006): Age-related macular degeneration. New Engl. J. Med, 355(14): 1474-85.

3. Bourla, D. H. and Young, T. A. (2008): AgeRelated Macula Degeration: A practical approach to a challenging disease. J. Am. Geriatr. Soc, 54(7):1130-5.

4. Esenwah, E. C. (2010): A Clinic based survey of Age- Related Macular Degeneration. J. Assoc. Nig. Optometric Educators. 3(2): 12-15

5. Age-Related Eye Disease Study Group. (2001): A randomized, placebo-controlled, clinical trial of high dose supplementation with vitamins $\mathrm{C}$ and $\mathrm{E}$, beta carotene, and zinc for age-related macular degeneration and vision loss: AREDS Report no. 8. Arch. Ophthalmol, 119:1417-36.

6. Friedman, D. S., O'Colmain, B. J. and Munoz, B. (2004): Prevalence of age-related macular degenerationin United States. Arch. Ophthalmol, 122:564-72.

7. Bressler, N. M., Bressler, S. B. and Congdon, N. G. (2003): Potential public health impact of Age-related Eye Disease Study results:
AREDS Report n. 11. Arch. Ophthalmol, 121:1621-4.

8. SanGiovanni, J. P., Chew, E. Y., Traci, E.C., Davis, M. D., Ferris III, F. L., Gensler, G. R., Kuriniji, N., Lindblad, S. A., Milton, R. C., Seddon, J. M. and Sperduto, R. D. (2007): The relationship of Dietry Lipid Intake and AgeRelated Macular Degeneration in a CaseControl Study. Arch. Ophthalmol, 125(5):671-9.

9. Gorin, M. B., Breitner, J. C., and De Jong, P. T. (1999): The genetics of Age-Related Macular Degeneration. Mol. Vis, 5:29.

10. Sarangarajan, R. (2005): Melanin aggregation and polymerization: possible implications in Age-relatedMacular Degeneration. Ophthalmic Res. 37:136-41.

11. Kanski, J.J. (1997): Age-Related Macular Degeneration. Clinical Ophthalmology. $3^{\text {rd }}$ Edn. Butterworth-Heinemann Publishers, Lincre House, Jordan Hill, Oxford, 514pp.

12. Glazer-Hockstein, C. and Dunaief, J. L. (2006): Could Blue-light blocking Lenses decrease the Risk of Age-realted Macular Degeneration? Retina, 26(1): 1-4.

13. Margrain, T. H., Boulton, M. and Marshall, J., Sliney, D. H.(2004): Do Blue Light filters confer protection against Age-related Macular Degeneration? Prog. Ret. Eye Res, 23(5): 52331 . 